\title{
Pattern of domiciliary consultations in the Trent region
}

\author{
ANNE I COUPLAND, GILLIAN B TODD
}

The variation in demand for domiciliary consultations between the specialties has been examined at a national level ${ }^{1}$ as requested by the review body on doctors' and dentists' remuneration. ${ }^{2}$ This paper looks at the distribution between specialties and areas within the Trent region of domiciliary consultations that were undertaken and accepted for payment in 1978-82. The information collected refers to the area health authorities in the Trent region before the reorganisation in 1982 .

The purpose and rules of operation of the domiciliary consultation service are set out in the terms and conditions of service for hospital and dental staff, ${ }^{3}$ which state that: "A domiciliary consultation shall . . . be understood to mean a visit to the patient's home, at the request of the general practitioner, and normally in his company, to advise on the diagnosis or treatment of a patient who on medical grounds cannot attend hospital." In addition, "Visits not falling within this definition include: a visit made at the instance of a hospital or specialist to review the urgency of a proposed admission to hospital or to continue to supervise treatment initiated or prescribed at a hospital or clinic." Visits to assess the domestic arrangements of patients in need of, or receiving, psychiatric or psychogeriatric treatment do not, therefore, comply with the regulations.

The information in this survey was collected for three reasons. Firstly, there is a duty to monitor the use of resources; secondly, the regional medical officer, and the district medical officer in a teaching district, authorises payment for domiciliary consultations and therefore requires information concerning the use of the service; and, thirdly, there are disparities in the demand for domiciliary consultations both between areas and between specialties.

Sheffield Health Authority, Westbrook House, Sheffield S11 8EU ANNE I COUPLAND, MB, CHB, registrar in community medicine

Trent Regional Health Authority, Fulwood House, Sheffield S10 3TH GILLIAN B TODD, MB, MFCM, specialist in community medicine

Correspondence to: Dr Anne I Coupland.

\begin{abstract}
Method of survey
The domiciliary consultation service in the Trent region was analysed for the period $1978-82$ by retrospectively scanning all the domiciliary claim forms submitted by consultants and accepted for payment in the region during October 1978 to September 1982. The information was analysed by area and specialty and compared with national figures.

Information about hospital resources was compiled from the hospital statistics for 1982 (SH3 forms). The number of domiciliary consultations, after being standardised by population served, was then compared with the number of beds and outpatient clinics available.
\end{abstract}

\section{Results}

Figure 1 shows the total number of domiciliary consultations per 1000 catchment population undertaken in each of the four years. There was a threefold difference between the highest and lowest. The three teaching areas had the lowest rate of domiciliary consultations with an average of $5 / 1000$ population a year, compared with the average for the non-teaching authority areas of $12 \cdot 6 / 1000$ population a year. When the non-teaching area with the highest rate (area 6) was excluded, however, the average for the nonteaching authority areas fell to $11 / 1000$ population a year.

Figure 2 shows the number of domiciliary consultations, beds, and outpatient clinics per 1000 catchment population in each of the eight area health authorities in the Trent region in 1981. When domiciliary consultations were compared with beds three groups of area health authorities emerged. The first group consisted of the three teaching area health authorities, with an average of $30 \%$ fewer domiciliary consultations than beds per 1000 population. The second group consisted of areas 4 and 5 , which were non-teaching, rural area health authorities with a similar pattern to the first group but with more domiciliary consultations and beds per 1000 population. The final group comprised three non-teaching, urban area health authorities (areas 6, 7, and 8), in which the number of beds per 1000 population was similar to that of the teaching authorities but the number of domiciliary consultations per 1000 population was almost double.

The number of outpatient clinics per 1000 population in each area was very similar, except in areas 1 and 8 ; area 1 had $25 \%$ more than the mean and area 8 had $25 \%$ fewer.

Figure 3 shows the number of domiciliary consultations undertaken in the Trent region in specialties in which the total annual number exceeded $1 \%$ of the total undertaken in the region in the year 1980-1. This shows the wide 
variation in demand for domiciliary consultations between specialties. Consultants in mental illness and geriatrics made the most domiciliary consultations, with mental illness accounting for $25 \%$ and geriatric medicine for $21 \%$ of the total. General medicine, general surgery, and orthopaedics made up $13 \%, 10 \%$, and $7 \%$ of the total, respectively.

Analysis of the number of consultations made by each consultant in each specialty (table I) showed that consultants in geriatric medicine carry out almost twice as many domiciliary consultations as consultants in mental illness because there were three times as many consultants in mental illness as in geriatric medicine. Similarly, consultants in chest medicine made twice as many consultations as those in general medicine. The average number of domiciliary consultations made by each consultant in the Trent region was similar to the national figure in several specialties. The most notable exceptions were chest medicine, mental illness, ophthalmology, and rheumatology and rehabilitation, in which there were at least $20 \%$ more

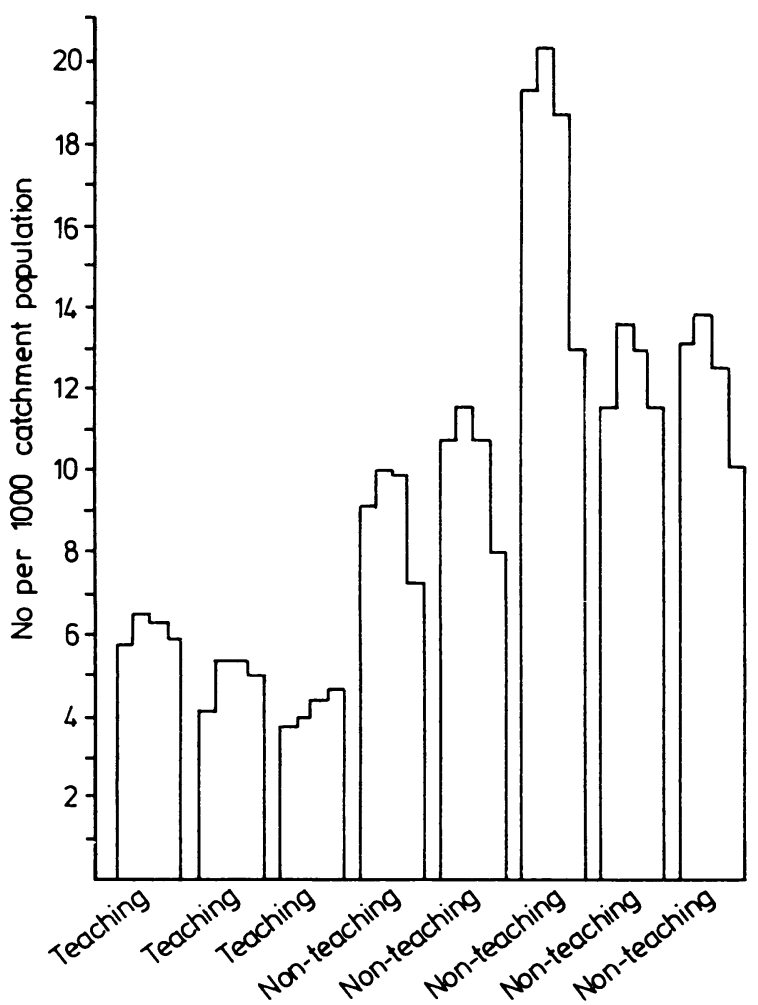

FIG 1-Number of domiciliary consultations per 1000 catchment population for each area in the Trent region, 1978-82.

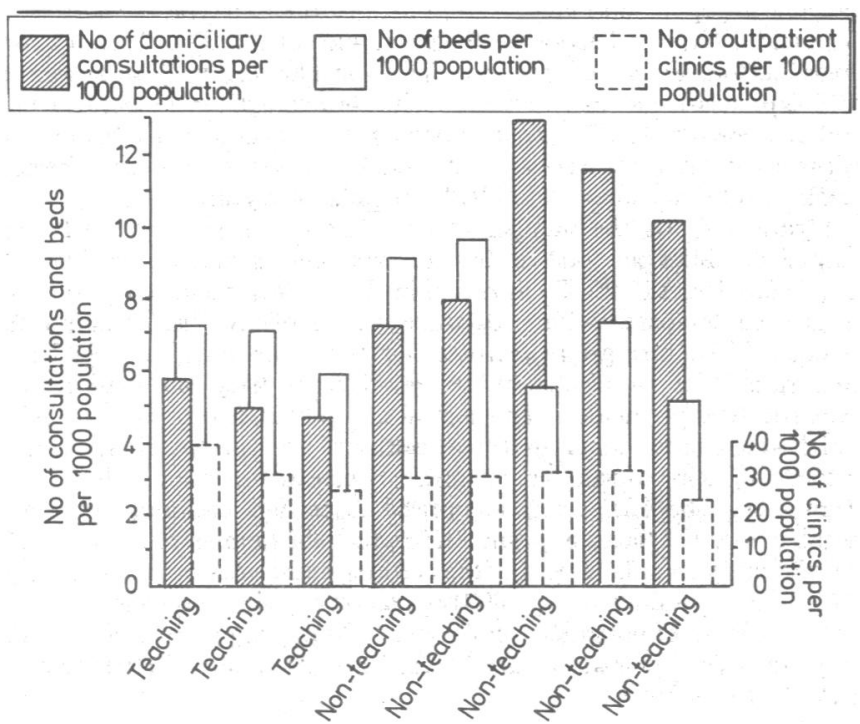

FIG 2--Comparison of number of domiciliary consultations with number of beds and outpatient clinics for each area in the Trent region during 1981.
TABLE I-Average number of domiciliary consultations per consultant by specialty in 1980-1

\begin{tabular}{|c|c|c|}
\hline & Trent region & England and Wales \\
\hline $\begin{array}{l}\text { Chest medicine } \\
\text { Dermatology } \\
\text { Ear, nose, and throat } \\
\text { General medicine } \\
\text { General surgery } \\
\text { Geriatrics } \\
\text { Haematology } \\
\text { Mental illness } \\
\text { Neurology and neurosurgery } \\
\text { Obstetrics and gynaecology } \\
\text { Ophthalmology } \\
\text { Orthopaedics } \\
\text { Paediatrics } \\
\text { Rheumatology and rehabilitation }\end{array}$ & $\begin{array}{r}105 \cdot 9 \\
60 \cdot 6 \\
13 \cdot 9 \\
48 \cdot 5 \\
52 \cdot 2 \\
211 \cdot 4 \\
19 \cdot 6 \\
110 \cdot 4 \\
28 \cdot 4 \\
12 \cdot 4 \\
39 \cdot 9 \\
46 \cdot 6 \\
13 \cdot 3 \\
68 \cdot 5\end{array}$ & $\begin{array}{r}47 \cdot 2 \\
60 \cdot 9 \\
16 \cdot 6 \\
71 \cdot 7 \\
51 \cdot 8 \\
211 \cdot 5 \\
23 \cdot 7 \\
92 \cdot 0 \\
33 \cdot 5 \\
17 \cdot 4 \\
22 \cdot 9 \\
43 \cdot 7 \\
22 \cdot 4 \\
53 \cdot 0\end{array}$ \\
\hline All specialties & 37.9 & 35.9 \\
\hline
\end{tabular}

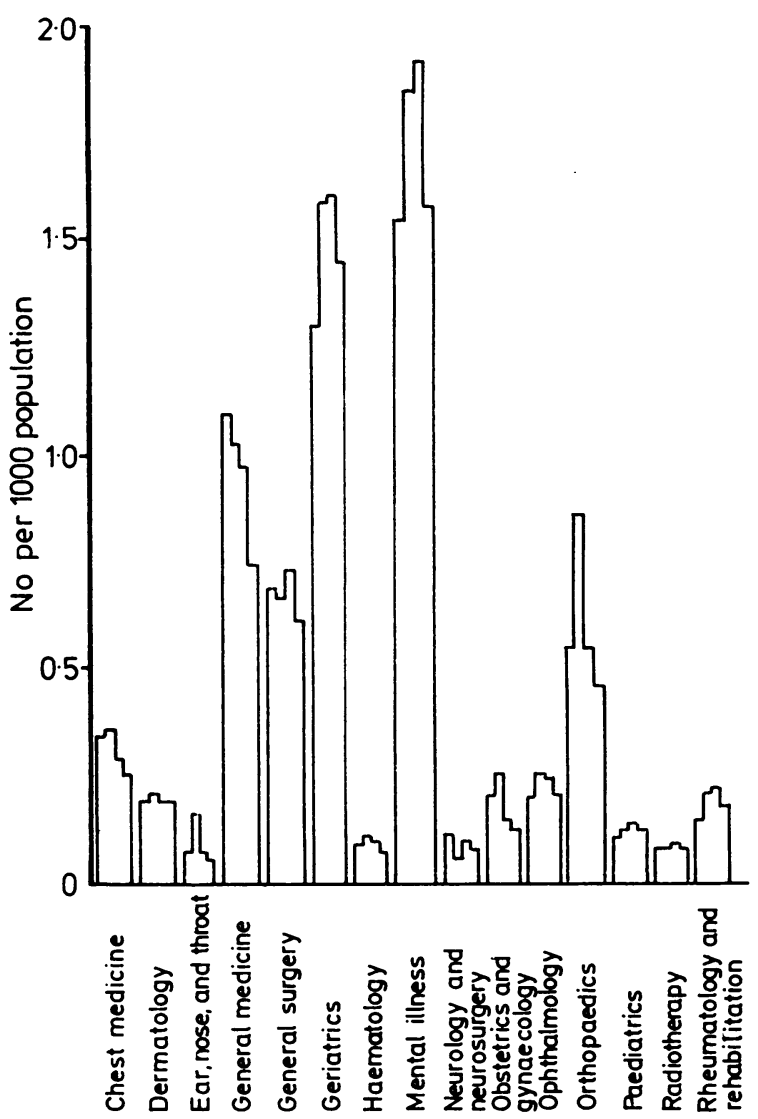

FIG 3-Number of domiciliary consultations per 1000 population in the Trent region according to specialty, 1978-82.

domiciliary consultations per consultant in the Trent region than the national average and general medicine, paediatrics, and obstetrics and gynaecology, in which there were at least $20 \%$ fewer.

Overall, consultants in all specialties in the Trent region in 1980 made on average 37.9 domiciliary consultations, compared with 35.9 in England and Wales. The Trent Region has $9 \cdot 2 \%$ of the population of England and Wales, and the total of 34500 domiciliary consultations in the region in 1980-1 was in fact $13 \%$ less than the expected figure of 39796 .

Table II shows the frequency distribution of domiciliary consultations performed by each consultant by specialty in 1980-1. This shows the variation in the number of domiciliary consultations made by consultants within a single specialty and indicates how misleading aggregated and averaged data can be. The mode in each specialty except for geriatrics was in the group of one to 49 domiciliary consultations, whereas in the specialty of geriatrics the mode of the distribution was in the group of 151-200.

Figure 4 shows the variation in the number of domiciliary consultations, beds, and outpatient clinics per 1000 popuiation in 1982 by specialty, and this reflects the different types of service provided. There were three distinct patterns: those specialties with fewer domiciliary consultations than beds; those with more domiciliary consultations than beds, but only in the range of $8-18 \%$ more; and those with many more domiciliary consultations than beds, in the range of $39-82 \%$ more.

These patterns were distinct and therefore any averages or comparisons of 
BRITISH MEDICAL JOURNAL VOLUME $290 \quad 11$ MAY 1985

aggregated data would be misleading. Account must be taken of the variation in the pattern of service between specialties, with some specialties being based more on an outpatient service while others have a high rate of emergency admissions. There are many explanations for the variable patterns - for example, in the specialty of geriatrics there were about three times as many domiciliary consultations as outpatient clinics, whereas in most of the other specialties there were $20-50 \%$ fewer domiciliary consultations than outpatient clinics. This was probably because the general debility of old age dictates the need for domiciliary consultations rather than attendance at an outpatient clinic. patterns of disease in the population, and to the experience of general practitioners, their desire to manage ill patients at home, and their need for expert help and guidance in doing so.

The wide variation in the number of domiciliary consultations undertaken by consultants in the same specialty is puzzling as it raises questions about the availability of resources and the health of individual communities. One reason put forward for the variation within specialties has been that in some specialties consultants prefer to see their patients as outpatients with the back up of

TABLE II-Distribution of consultants by number of domiciliary consultations performed and by specialty in 1980-1

\begin{tabular}{|c|c|c|c|c|c|c|c|c|c|}
\hline & \multicolumn{9}{|c|}{ No of visits } \\
\hline & $1-50$ & $51-100$ & $101-50$ & $151-200$ & $201-50$ & $251-300$ & $301-50$ & $351-400$ & $401-50$ \\
\hline Chest medicine & 8 & 3 & 1 & 2 & 1 & & & & \\
\hline Dermatology & 6 & 2 & i & i & $\mathrm{i}$ & & & & \\
\hline Ear, nose, and throat & 23 & 1 & $x^{*}$ & $x^{2}$ & & & & & \\
\hline General medicine & 18 & 15 & 5 & 7 & 3 & 1 & & & \\
\hline General surgery & 38 & 13 & 2 & 5 & 1 & & & 1 & \\
\hline Geriatrics & 1 & & 4 & 8 & 2 & 6 & 4 & 2 & 2 \\
\hline Haematology & 14 & & & & 1 & & & & \\
\hline Mental illness & 25 & 17 & 10 & 9 & 6 & 7 & 4 & 1 & \\
\hline Neurology and neurosurgery & 6 & 3 & 1 & & & & & & \\
\hline Obstetrics and gynaecology & 24 & 2 & 1 & 1 & & & & & \\
\hline Ophthalmology & 18 & 2 & 4 & i & & & & & \\
\hline Orthopaedics & 25 & 6 & 4 & & 2 & 3 & & & \\
\hline Paediatrics & 23 & & 1 & & 1 & & & & \\
\hline Radiology & 15 & 2 & & & & & & & \\
\hline Rheumatology and rehabilitation & 6 & 4 & 1 & 1 & & 1 & & & \\
\hline
\end{tabular}

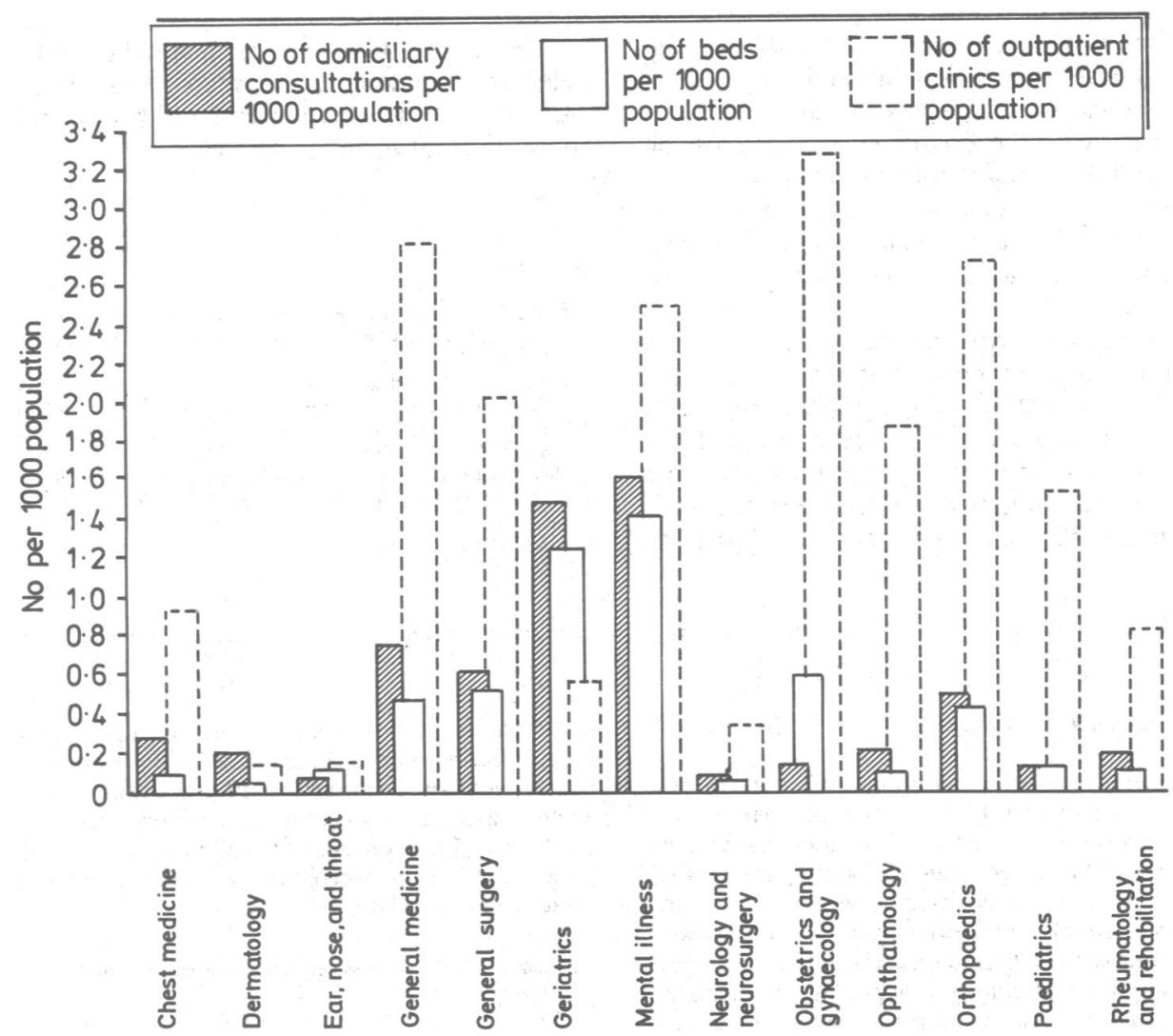

FIG 4 - Comparison of number of domiciliary consultations with number of beds and outpatient clinics for each specialty.

\section{Discussion}

The domiciliary consultation service was included in the National Health Service Act of 1946 as a free service to general practitioners. In 1955 consultants were paid to provide this service. ${ }^{4}$ In the Annis Gillie report in 1963 it was decided that this service was not only to provide advice on diagnosis or treatment but also to aid joint consultation between the consultant and the general practitioner. ${ }^{5}$ If the service meets that need the demand should be related to the investigatory equipment and so discourage requests for domiciliary consultations from general practitioners.

It is also difficult to explain, without thorough examination, the twofold difference in demand for domiciliary consultations between teaching and non-teaching authorities. This might be an indication of the relation of domiciliary consultations to hospital resources, not to patients' needs. It may be that consultants in teaching authorities are less keen to undertake such visits because they find it easier to treat patients in hospital with a full range of investigative equipment 
available. In the Trent region the domiciliary claim torms indicate that many patients are admitted directly as a result of such consultations. Detailed analysis of the domiciliary consultations in the specialties of mental illness and geriatrics in one area showed that $32 \%$ of psychiatric and $64.7 \%$ of geriatric domiciliary consultations resulted in an immediate admission, which perhaps indicates that the service is used as a screening service for admissions rather than to provide advice to general practitioners.

If the service in some specialties is used as a screening service for admissions then perhaps such visits should be part of the contractual commitments of the consultants. General practitioners often prefer geriatric patients to be assessed in their own home so that sensible plans can be made for when they are discharged. Because of this home assessment sessions in the Trent region have been included in the contracts of consultants in geriatric medicine since 1972. Perhaps, in view of the large number of domiciliary consultations in psychiatry, this should be extended to mental illness.

In theory, the availability of beds or outpatient appointments should not alter the rate of domiciliary consultations; in practice, however, it is likely to do so if it is the only way a patient can be seen within a few days. The two rural, non-teaching authorities (areas 4 and 5) had more beds and a higher number of domiciliary consultations per 1000 population than the other areas. Hospital resources therefore cannot completely explain the imbalance in the rates of domiciliary consultation.

Dowie suggested that with an increase in consultants there would be a corresponding increase in the amount of domiciliary work, 'but as a domiciliary consultation is justified only on medical grounds the rate of domiciliary consultation should increase only if the population increases or the overall health of the population declines. Such suggestions therefore cause concern and require investigation.

Consultants in most specialties in the Trent region undertook, on average, the same number of domiciliary consultations as consultants in England and Wales. There were, however, a few exceptions, and further study may be needed in these specialties to see if there is any epidemiological explanation for this or if it is a manifestation of differing clinical practices.

We conclude that a review of the present rules under which a domiciliary consultation is undertaken is necessary. In addition, the variation between specialty, consultant, and geographic area needs to be fully understood when the domiciliary service is regarded as a source of income by the doctors' and dentists' review body. The domiciliary consultation service is important to the general practitioner so that he can obtain specialist advice on the care of ill patients at home. Ease of availability is a vital part of the service and must be preserved if a review of the present rules is ever undertaken. We suggest that all consultants in mental illness and geriatric medicine should have sessions for home assessment in their contracts. The relation between domiciliary consultations and outpatient accessibility needs to be examined in more detail. It would also be interesting to establish whether an increase in the number of consultant outpatient clinics in the community reduces the need for domiciliary consultations.

The attitude and patterns of referral of general practitioners could also explain some of the variation seen. The mobility of patients, not just their age, also has to be taken into consideration in some specialties. The prevalence of certain diseases or conditions may be higher in some geographical areas, leading to an increased rate of referral, and so variations should be investigated where appropriate by a thorough study of prevalence. In one district in the Trent region a high proportion of domiciliary consultations in two specialties was attributable to the diagnoses of rheumatoid arthritis, prolapsed intervertebral disc, and deep vein thrombosis.

When aggregated data are used the pattern of domiciliary consultations in the Trent region is similar to that in England and Wales. When the data are examined in a disaggregated form then there is a large variation not only between specialties but, more importantly, within specialties-that is, between consultants. This shows the importance of careful examination of aggregated data.

Three patterns emerged when the number of domiciliary consultations was compared with the number of beds and outpatient clinics. These patterns should be taken into account when services are being planned.

We believe that a study should be made into the patterns of referral of general practitioners requesting domiciliary consultations and the information analysed before and after standardisation for local facilities and resources.

\section{References}

1 Dowie R. National trends in domiciliary consultations. Br Med f 1983;286:819-22.

2 Review Body on Doctors' and Dentists' Remuneration. Twelfth report. London: HMSO, 1982. (Cmnd 8550 .)

3 Department of Health and Social Security. National Health Service hospital medical and dental staff England and Wales) terms and conditions of service, April 1981, revised. London: HMSO, 1981. 4 Sheffield Regional Hospital Board. Quinquennial report to Sheffeld Regional Hospital Board. Sheffield: Sheffield Regional Hospital, 1952:57.

5 Subcommittee of the Standing Medical Advisory Committee of the Central Health Service Council. The field of work of the family doctor. London: HMSO, 1963.

(Accepted 8 fanuany 1985)

\section{Is there any evidence of an association between incest and anorexia nervosa?}

It is difficult to point to a general association between incest and anorexia nervosa. The cause of anorexia nervosa is said to be multifactorial, ' a view that is partly an admission of ignorance and partly a recognition that various factors may interact to bring about the illness in an adolescent girl or young woman. Anorexia nervosa is often preceded by a traumatic event-for instance, a bereavement, physical illness, or parental separation. It is usually accompanied by sexual immaturity as well as amenorrhoea, the latter and possibly the former being associated with impaired hypothalamic function. The patient's sexual conflicts and difficulties will probably have a psychogenesis, the source of which should be sought for and might lie, for example, within her family. The interaction between family members should then be explored: a close and seductive relationship is sometimes found to exist between the patient and her father. It is uncertain how often actual sexual contact occurs within such a relationship.

The frequency of incest in the general population is unknown but it is not rare. There are no controlled studies of the consequences of incest involving children. ${ }^{2}$ A recent report suggests that a history of adverse sexual experiences, including occasional incestuous relationships, may be elicited in as many as two thirds of patients with anorexia nervosa ( $R$ Oppenheimer, paper at conference on anorexia nervosa, Swansea, 1984). If such a patient discloses a past incestuous relationship this should be taken seriously in her management. The experience has probably given rise to adverse feelings about her femininity which may be expressed through an excessive concern with body shape and size, harsh dieting, and, eventually, anorexia nervosa. Hence she should be encouraged to express her feelings about these events which may have been disclosed for the first time. It should be possible to explore with the patient an association between her conflicts over her sexuality and her concern with body size. This should form part of a general programme of management for anorexia nervosa. ${ }^{3}$-GERALD RUSSELL, professor of psychiatry, London.

1 Garfinkel PE, Garner DM. Anorexia nervosa, a multidimensional perspective. New York: Brunner/Mazel Inc, 1982:188-213.

2 Wolff S. Incest (child referred to psychiatrist). In: Russell GFM, Hersov LA, eds. Handbook of psychiaty. Vol 4. Cambridge: Cambridge University Press, 1983;356-8

3 Russell GFM. Anorexia nervosa and bulimia nervosa. In: Russell GFM, Hersov LA, eds. Handbook of psychiatry. Vol 4. Cambridge: Cambridge University Press, 1983;285-98.

Rhesus isoimmunisation may occur during labour or as a consequence of interference with the adherence of the placenta during late pregnancy. At what stage in a pregnancy does the risk of isoimmunisation become significant?

Appreciable leakage of fetal cells into the maternal circulation giving rise to rhesus isoimmunisation is most likely to occur during the third stage of labour. Nevertheless, fetomaternal haemorrhage can occur during the antenatal period, although primary immunisation before the 28th week of pregnancy is rare.-G J LEWIS, consultant obstetrician and gynaecologist, Stourbridge. 